\title{
A Variscan continental collision of the West Sudetes and the Brunovistulian terrane: a contribution from structural and metamorphic record of the Stronie Formation, the Orlica-Śnieżnik Dome, SW Poland
}

\author{
Mirosław Jastrzębski
}

Published online: 28 October 2008

(c) Springer-Verlag 2008

\section{Erratum to: Int J Earth Sci (Geol Rundsch) DOI 10.1007/s00531-008-0357-5}

It is regretted that following geochronological data have been omitted in Table 1. Please find the corrected table below.

\begin{tabular}{ccllc}
\hline Localisation & Age (Ma) & Method & $\begin{array}{l}\text { Interpre- } \\
\text { tation }\end{array}$ & Reference \\
\hline \multicolumn{2}{c}{ Orthogneisses and migmatites } & & & \\
Średniak Mt. & $336.3 \pm 3.5$ & Rb-Sr, WR-Ms & Cooling & Lange et al. \\
& $331.3 \pm 3.3$ & Rb-Sr, WR-Bt & Cooling & (2005b) \\
Nowa Wieś & $339.6 \pm 3.9$ & Rb-Sr, WR-Ms & Cooling & \\
Nowa Wieś & $341.6 \pm 3.7$ & Rb-Sr, WR-Ms & Cooling & \\
& $333.8 \pm 3.4$ & Rb-Sr, WR-Bt & Cooling & \\
Przednia Mt. & $336.1 \pm 3.5$ & Rb-Sr, WR-Ms & Cooling & \\
& $332.0 \pm 3.4$ & Rb-Sr, WR-Bt & Cooling & \\
Wrzosówka & $335.5 \pm 3.5$ & Rb-Sr, WR-Ms & Cooling & \\
& $330.4 \pm 3.4$ & Rb-Sr, WR-Bt & Cooling & \\
Lądek Zdrój- & $336.8 \pm 3.6$ & Rb-Sr, WR-Ms & Cooling & \\
Radochów & $327.3 \pm 3.3$ & Rb-Sr, WR-Bt & Cooling & \\
Międzygórze & $331.3 \pm 3.4$ & Rb-Sr, WR-Bt & Cooling & \\
Kuźnicza & $334.1 \pm 3.5$ & Rb-Sr, WR-Ms & Cooling & \\
Góra Mt. & $318.8 \pm 3.2$ & Rb-Sr, WR-Bt & Cooling & \\
Międzygórze & $329.8 \pm 3.4$ & Rb-Sr, WR-Bt & Cooling \\
\hline
\end{tabular}

The online version of the original article can be found under doi:10.1007/s00531-008-0357-5.

M. Jastrzębski ( $₫)$

Institute of Geological Sciences, Polish Academy of Sciences, Podwale 75, 50-449 Wrocław, Poland

e-mail:mjast@interia.pl

\begin{tabular}{lllll}
\hline Localisation & Age (Ma) & Method & $\begin{array}{l}\text { Interpre- } \\
\text { tation }\end{array}$ & Reference \\
\hline Stojków & $339.9 \pm 3.8$ & Rb-Sr, WR-Ms & Cooling \\
& $336.9 \pm 3.4$ & Rb-Sr, WR-Bt & Cooling \\
Trojak Mt. & $334.1 \pm 3.5$ & Rb-Sr, WR-Ms & Cooling \\
& $318.7 \pm 3.2$ & Rb-Sr, WR-Bt & Cooling \\
Nowa Morawa & $330.4 \pm 3.5$ & Rb-Sr, WR-Ms & Cooling \\
Strachocin & $364-341$ & U-Pb, Zrn- & Metamorphism \\
& \multicolumn{3}{c}{ SHRIMP } \\
\hline
\end{tabular}

\section{Reference}

Lange U, Bröcker M, Armstrong R, Żelaźniewicz A, Trapp E, Mezger K (2005b) The orthogneisses of the Orlica-Śnieżnik complex (West Sudetes, Poland): geochemical characteristics, the importance of pre-Variscan migmatization and constraints on the cooling history. J Geol Soc 162:973-984 